\title{
Global structure and one-sign solutions for second-order Sturm-Liouville difference equation with sign-changing weight
}

\author{
fumei $\mathrm{ye}^{1}$
}

${ }^{1}$ Northwest Normal University

December 21, 2020

\begin{abstract}
This paper is devoted to study the discrete Sturm-Liouville problem $\$ \$ \backslash$ left $\backslash\{\backslash$ begin $\{$ array $\}\{11\}-\backslash$ Delta $(\mathrm{p}(\mathrm{k}) \backslash$ Delta $\mathrm{u}(\mathrm{k}-$ 1) $)+\mathrm{q}(\mathrm{k}) \mathrm{u}(\mathrm{k})=\backslash \operatorname{lambda} \mathrm{m}(\mathrm{k}) \mathrm{u}(\mathrm{k})+\mathrm{f}_{-} 1(\mathrm{k}, \mathrm{u}(\mathrm{k}), \backslash$ lambda $)+\mathrm{f}_{-} 2(\mathrm{k}, \mathrm{u}(\mathrm{k}), \backslash$ lambda $), \backslash \backslash \mathrm{k} \backslash \operatorname{in}[1, \mathrm{~T}] \_\mathrm{Z}, \backslash \backslash[2 \mathrm{ex}] \mathrm{a} \_0 \mathrm{u}(0)+\mathrm{b} \_0 \backslash$ Delta $\mathrm{u}(0)=0, \backslash$ a_1u $(\mathrm{T})+$ b_$_{-} \backslash$ Delta $\mathrm{u}(\mathrm{T})=0$, $\backslash$ end $\{$ array $\} \backslash$ right. $\$ \$$ where $\$ \backslash$ lambda $\backslash$ in $\backslash$ mathbb $\{\mathrm{R}\} \$$ is a parameter, $\$ f_{-} 1, \mathrm{f}_{-} 2 \backslash$ in $\mathrm{C}\left([1, \mathrm{~T}]_{-}-\right.$ $\mathrm{Z} \backslash$ times $\left.\backslash \operatorname{mathbb}\{\mathrm{R}\}^{\wedge} 2, \backslash \operatorname{mathbb}\{\mathrm{R}\}\right) \$, \$ \mathrm{f}_{-} 1 \$$ is not differentiable at the origin and infinity. Under some suitable assumptions on nonlinear terms, we prove the existence of unbounded continua of positive and negative solutions of this problem which bifurcate from intervals of the line of trivial solutions or from infinity, respectively.
\end{abstract}

\section{Hosted file}

second-order indefinite weight difference equation.pdf available at https://authorea.com/ users/371679/articles/499865-global-structure-and-one-sign-solutions-for-second-ordersturm-liouville-difference-equation-with-sign-changing-weight 